\title{
Case Report: Successful DaVinci-Assisted Major Liver Resection for Alveolar Echinococcosis
}

\section{Mohammad Golriz ${ }^{1}$, Viktoria Flossmann ${ }^{1}$, Ali Ramouz ${ }^{1}$, Ali Majlesara ${ }^{1}$, Yakup Kulu ${ }^{1}$, Marija Stojkovic ${ }^{2}$ and Arianeb Mehrabi ${ }^{1 *}$}

${ }^{1}$ Department of General, Visceral, and Transplantation Surgery, University of Heidelberg, Heidelberg, Germany, ${ }^{2}$ Department of Clinical Tropical Medicine, University of Heidelberg, Heidelberg, Germany

OPEN ACCESS

Edited by:

Marcos Vinicius Perini,

The University of Melbourne, Australia

Reviewed by:

Andrew Gumbs,

Centre Hospitalier Intercommunal de

Poissy, France

Mahir Gachabayov,

Westchester Medical Center,

United States

*Correspondence:

Arianeb Mehrabi

Arianeb.Mehrabi@

med.uni-heidelberg.de

Specialty section:

This article was submitted to Visceral Surgery,

a section of the journal

Frontiers in Surgery

Received: 08 December 2020 Accepted: 03 February 2021

Published: 04 March 2021

Citation:

Golriz M, Flossmann V, Ramouz A,

Majlesara A, Kulu Y, Stojkovic M and

Mehrabi A (2021) Case Report:

Successful DaVinci-Assisted Major

Liver Resection for Alveolar

Echinococcosis

Front. Surg. 8:639304.

doi: 10.3389/fsurg.2021.639304
We report a case of successful robot-assisted major liver resection in a patient with liver alveolar echinococcosis (AE). A 62-year-old male patient was incidentally diagnosed with a large infiltrative lesion in the right liver lobe suspicious for $A E$. A radical surgical resection as a right-sided hemihepatectomy was indicated. The operation was carried out via a robotic-assisted procedure using the DaVinci Xi Surgical System. The tumor measured $12.4 \times 8.8 \mathrm{~cm}$ and was successfully resected through a suprapubic incision of $13 \mathrm{~cm}$. The patient was free of pain after the second post-operative day. A fluid collection near the resection plate was easily drained without bile leakage. The patient had no surgical complications. Radical resection is inevitable for adequate curative therapy of $A E$ and provides clear margins. Robotic surgery is a relatively new and safe option for curative resection of $\mathrm{AE}$ lesions, with remarkable advantages for patients and surgeons.

Keywords: alveolar echinococcosis, robotic surgery, major liver resection, DaVinci-assisted major liver resection, hemihepatectomy

\section{INTRODUCTION}

Alveolar echinococcosis (AE), caused by Echinococcus (E.) multilocularis, is widely distributed in the northern hemisphere. Other species are E. granulosus leading to cystic echinococcosis, and E. vogeli and E. oligarthrus, causing polycystic echinococcosis (1).

In Europe, the prevalence of $\mathrm{AE}$ is low and is estimated at 0.64/100,000 in Germany. Most infections exclusively affect the liver. In patients with very large liver masses and invasion of major hepatic vessels metastases to the lung and less frequently to the brain may occur. There are two treatment options: (1) parasitostatic treatment with benzimidazoles, which is indicated in cases where curative resection is not possible or suitable, and (2) radical resection with safety margins of $2 \mathrm{~cm}$. However, safety margins of $1 \mathrm{~mm}$ have also been tolerated without recurrence of disease $(2-5)$. Following an R0 resection, albendazole treatment is recommended for at least 2 years. Patients have to be followed for 10 years after surgical treatment. We report here in detail a case of robot-assisted major liver resection for alveolar echinococcosis treatment. 


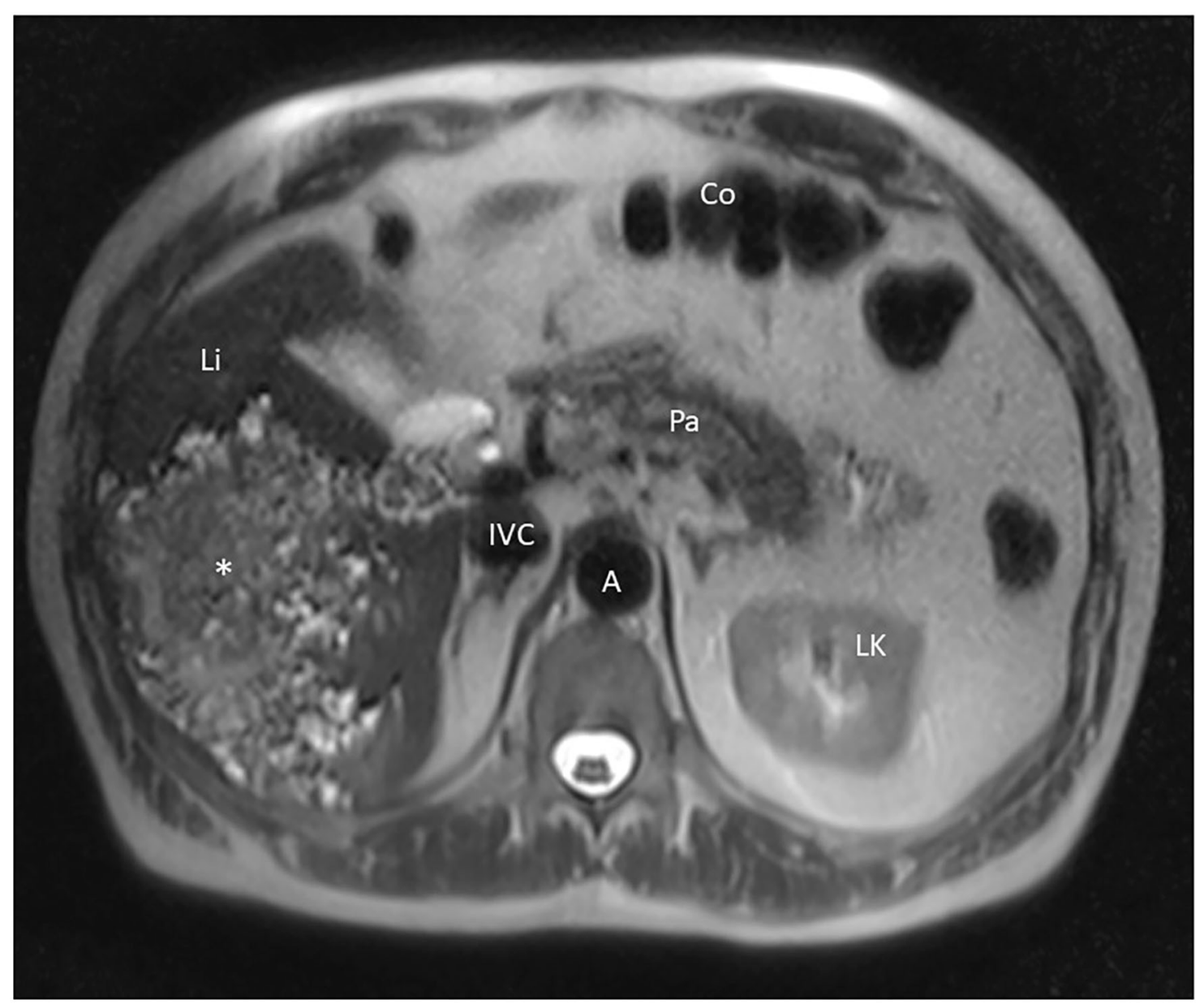

FIGURE 1 | MRI imaging showing the AE lesion of our patient in the axial plane. Li, liver; Co, colon; Pa, pancreas; LK, left kidney; A, aorta; IVC, inferior vena cava, ${ }^{\star} \mathrm{AE}$ tumor.

\section{CASE DESCRIPTION}

A 62-year-old male patient, incidentally diagnosed with a large infiltrative lesion in the liver during trauma clarification, presented himself in our hospital. An abdominal CT scan was performed to rule out trauma injuries and showed a poorly defined lesion in the right liver lobe suspicious for cholangiocellular carcinoma or alveolar echinococcosis (AE). The patient denied any abdominal or B symptoms, and there was no family history of malignant disease. He lived in a rural area in Germany.

For further clarification, blood tests and an MRI scan of the abdomen were performed. Echinococcus serology was positive (E. species 1.541 optical density with a cut-off value of 0.988 and $E$. multilocularis 1.666 optical density with a cut-off value of 0.625 ). Immunoblot revealed 7-, 16-, $18-$, and $26-28 \mathrm{kDa}$ proteins in

Abbreviations: AE, alveolar echinococcosis; E, Echinococcus; MRI, magnetic resonance imaging; Li, liver; Co, colon; $\mathrm{Pa}$, pancreas; LK, left kidney; A, aorta; IVC, inferior vena cava. size. Blood tumor markers were in the normal range (CEA: 0.7 ug/l, CA 19-9: $23.4 \mathrm{U} / \mathrm{ml}$, and AFP: $2.1 \mathrm{IU} / \mathrm{ml}$ ). MRI imaging showed an enormous manifestation of $\mathrm{AE}$ in the right liver with microcystic and necrotic components that contacted the right pedicle (Figure 1); no further suspicious lesions were present in the liver or lymph nodes. There were no distant metastases (head, chest). Our multidisciplinary liver board discussed the case and recommended radical surgical resection of the lesion with a right-sided hemihepatectomy and parasitostatic treatment with albendazole.

The operation was carried out as a robotic-assisted procedure using the DaVinci Xi Surgical System (Intuitive Surgical Inc., United States). The tumor measured $12.4 \times 8.8 \mathrm{~cm}$ (Figure 2a) and was successfully removed from the abdomen through a suprapubic incision of $13 \mathrm{~cm}$ (Figure 2b). Histopathological examinations revealed clear resection margins. The patient was mobile on the first post-operative day and was free of pain after the second post-operative day. Postoperatively, a fluid collection was observed near the resection plate that was easily drained without bile leakage. The patient had 


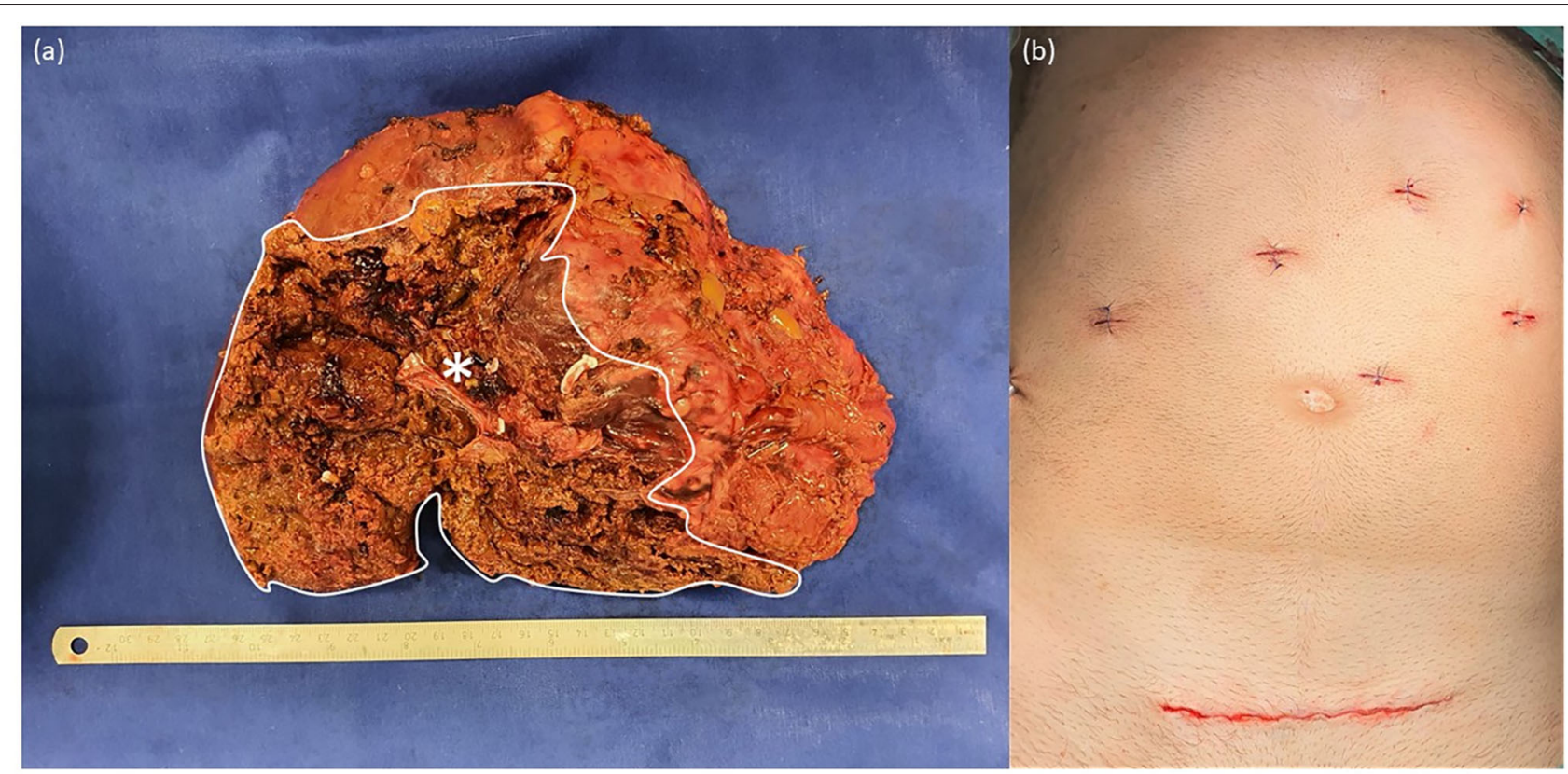

FIGURE 2 | (a) Right hemi-liver including the tumor measuring $12.4 \times 8.8 \mathrm{~cm}$ as well as segments 5, 6, 7, and 8. *AE tumor. (b) Postoperative scars after DaVinci-assisted right hemihepatectomy.

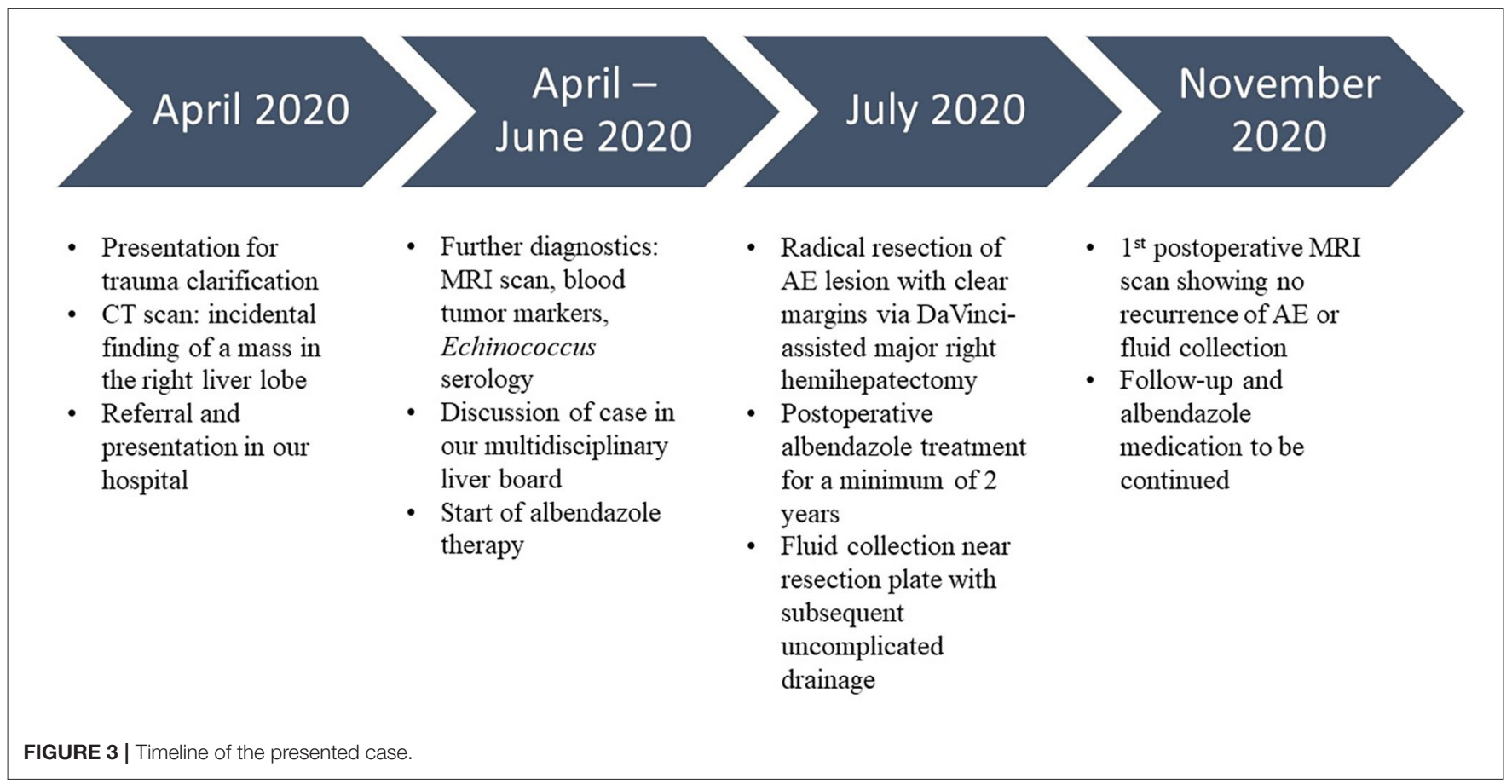

no surgical complications such as posthepatectomy biliary leakages or bleeding. Albendazole therapy was prescribed for 2 years after surgery, and follow-up examinations were scheduled. During the first 6 months of follow-up, the MRI scan showed no recurrent $\mathrm{AE}$ or fluid collection at the resection plate, and E. species serology was negative. The patient presented in a good general state of health and reported good recovery and return to daily activities and work. The timeline of the described case is summarized in Figure 3. 


\section{DISCUSSION}

For successful treatment of AE, an expert consensus recommends a multidisciplinary discussion to plan the therapeutic strategy according to the stage of the disease. Preoperative treatment with parasitocidal agents is not routinely suggested in management of AE. However, it has been suggested to stabilize the progression of the disease with parasitocidal agents, which was administered in our patient (6). Radical surgery should be the treatment of choice in all patients where feasible and carried out in line with oncological standards leaving tumor-free margins of $2 \mathrm{~cm}$. However, margins of $1 \mathrm{~mm}$ have also been tolerated without recurrence of the disease. Postoperatively, drug treatment with the benzimidazoles, an anthelmintic agent, for at least 2 years in $\mathrm{R} 0$-resection patients, or even for life in the case of incomplete resection, is recommended. The drug is usually administered at a daily dose of $800 \mathrm{mg}$, divided into 2 doses. After surgery and the start of benzimidazole therapy, e.g., with albendazole, surveillance for 10 years to detect possible recurrence is strongly advised. In palliative situations, interventional procedures are to be favored over palliative surgery, which is to be avoided in any situation possible (2-5).

Minimally invasive surgical procedures can improve post-operative mobilization and reduce wound-healing impairment compared with open surgery (7). Robotic-assisted surgery is widely used as a replacement for conventional laparoscopic surgery because of its enhanced precision (8), dexterity, and intuitiveness $(9,10)$. The crucial benefits of robotic surgery are lower overall morbidity rates and a shorter hospital stay than open surgery (10-13). Reduced post-operative pain $(10,12)$ and better cosmetic results (11) also improve patients' outcomes after robotic surgery. Robotic assistance systems, such as DaVinci $\mathrm{Xi}$, can help overcome the disadvantages of conventional laparoscopic surgery by giving surgeons a better view of the operating field (high-definition, 3-dimensional vision with adjustable magnification) and by stabilizing the surgical instruments with an extended range of motion $(7,10,14,15)$. Additionally, the operating position is more comfortable for the surgeons, preventing fatigue, improving concentration, and reducing stress $(10,16)$. Despite scarce evidence for robotic liver surgery (7), liver resection safety and feasibility by robotic-assisted procedures have been well-demonstrated $(8,13-15)$. AE is not a malignant disease but needs to be surgically treated as such. The robotic system offered AE patients a safe tumor resection with clear margins, early post-operative mobilization, pain-free recovery, and optimal cosmetic results and provided surgeons with a stress-free and comfortable operating environment.

In the literature, several cases have been reported to undergo robot-assisted treatment of hepatic echinococcosis. Of these, most reports have focused on the approach to patients with cystic echinococcosis, which its management differs from patients with $\mathrm{AE}$. In these patients, total cystectomy, minor, and major liver resections have been mostly utilized, as reported by Magistri et al. (17) and Felsenreich et al. (18). Conversely, few patients with $\mathrm{AE}$ have been reported to undergo liver resection using robotic approaches, especially major liver resection. Although Efanov have addressed the safety and feasibility of robot-assisted liver resection in a patient with $\mathrm{AE}$, a bisegmentectomy was indicated for the patient rather than a major liver resection (19). Zhao et al. reported 5 cases, of which only 1 patient presents with $\mathrm{AE}$; all other patients are diagnosed with cystic echinococcosis (20). This single case with $\mathrm{AE}$ underwent a right hemihepatectomy. Therefore, our report is one of the very first reports of roboticassisted major liver resection for AE treatment. Of course, there are limitations with robotic surgery for AE, such as abdominal adhesions or lack of surgical experience, and careful evaluation of each case is necessary.

Rupture is a rare complication in $\mathrm{AE}$ and has only been described once in the literature due to trauma (21). The ruptured lesion was managed via repeated resections and albendazole medication. Although the intraoperative rupture of hepatic AE has not been reported, precautions should be taken during the operation and the resection has to be performed like a malignant tumor. For major hepatectomy, subcostal, mid-line, or suprapubic incisions can be performed for the safe removal of the tumor. Since subcostal incisions have been associated with a higher incidence of post-operative pulmonary complications and mid-line incisions carry a higher risk of developing postoperative incision hernia (22), a suprapubic incision seemed to be the best option. Additionally, suprapubic incisions lead to a better cosmetic result (23-25).

\section{CONCLUSION}

In conclusion, $\mathrm{AE}$ is a rare disease that should be considered a differential diagnosis in patients with benign and malignant liver tumors. For adequate curative therapy, AE requires radical resection with clear margins and post-operative anti-parasitic drug treatment. Robotic surgery is a relatively new but safe option for curative resection of $\mathrm{AE}$ lesions with benefits for patients and surgeons.

\section{DATA AVAILABILITY STATEMENT}

The raw data supporting the conclusions of this article will be made available by the authors, without undue reservation.

\section{ETHICS STATEMENT}

Ethical review and approval was not required for the study on human participants in accordance with the local legislation and institutional requirements. The patients/participants provided their written informed consent to participate in this study. Written informed consent was obtained from the individual(s) for the publication of any potentially identifiable images or data included in this article. 


\section{AUTHOR CONTRIBUTIONS}

MG, VF, AR, and AMa prepared and wrote this article. $\mathrm{MG}, \mathrm{AR}$, and AMe were involved in managing the patient besides preparing the intraoperative pictures. MG, YK, MS,

\section{REFERENCES}

1. Akbulut S. Parietal complication of the hydatid disease: comprehensive literature review. Medicine. (2018) 97:e10671. doi: 10.1097/MD.0000000000010671

2. Brunetti E, Kern P, Vuitton DA, Echinococcosis WPftW-IWGo. Expert consensus for the diagnosis and treatment of cystic and alveolar echinococcosis in humans. Acta Trop. (2010) 114:1-16. doi: 10.1016/j.actatropica.2009.11.001

3. Chen KF, Tang YY, Wang R, Fang D, Chen JH, Zeng Y, et al. The choose of different surgical therapies of hepatic alveolar echinococcosis: a single-center retrospective case-control study. Medicine. (2018) 97:e0033. doi: 10.1097/MD.0000000000010033

4. Hillenbrand A, Gruener B, Kratzer W, Kern P, Graeter T, Barth $\mathrm{TF}$, et al. Impact of safe distance on long-term outcome after surgical therapy of alveolar echinococcosis. World J Surg. (2017) 41:1012-8. doi: 10.1007/s00268-016-3813-6

5. Kamiyama T. Recent advances in surgical strategies for alveolar echinococcosis of the liver. Surg Today. (2019) 50:13607. doi: 10.1007/s00595-019-01922-6

6. Salm LA, Lachenmayer A, Perrodin SF, Candinas D, Beldi G. Surgical treatment strategies for hepatic alveolar echinococcosis. Food Waterborne Parasitol. (2019) 15:e00050. doi: 10.1016/j.fawpar.2019.e00050

7. Kirchberg J, Weitz J. Evidenz der Roboter-Chirurgie in der onkologischen Viszeralchirurgie. Der Chirurg. (2019) 90:37986. doi: 10.1007/s00104-019-0812-9

8. Ban D, Ishikawa Y, Tanabe M. Can robotic liver resection compensate for weaknesses of the laparoscopic approach? Hepatobiliary Surg Nutr. (2020) 9:385-7. doi: 10.21037/hbsn.2019.11.02

9. Chen Y, Zhang Sa, Wu Z, Yang B, Luo Q, Xu K. Review of surgical robotic systems for keyhole and endoscopic procedures: state of the art and perspectives. Front Med. (2020) 14:382-403. doi: 10.1007/s11684-020-0781-x

10. Medical Advisory S. Robotic-assisted minimally invasive surgery for gynecologic and urologic oncology: an evidence-based analysis. Ont Health Technol Assess Ser. (2010) 10:1-118.

11. Barbosa Joao ABA, Barayan G, Gridley Chad M, Sanchez Daniela CJ, Passerotti Carlo C, Houck Constance S, et al. Parent and patient perceptions of robotic vs. open urological surgery scars in children. J Urol. (2013) 190:24450. doi: 10.1016/j.juro.2012.12.060

12. Darr C, Cheufou D, Weinreich G, Hachenberg T, Aigner C, Kampe S. Robotic thoracic surgery results in shorter hospital stay and lower postoperative pain compared to open thoracotomy: a matched pairs analysis. Surg Endosc. (2017) 31:4126-30. doi: 10.1007/s00464-017-5464-6

13. Machairas N, Papaconstantinou D, Tsilimigras DI, Moris D, Prodromidou A, Paspala A, et al. Comparison between robotic and open liver resection: a systematic review and meta-analysis of short-term outcomes. Updat Surg. (2019) 71:39-48. doi: 10.1007/s13304-019-00629-0

14. Croner R, Franz M, Arend J, Rahimli M, Stockheim J, Negrini VR, et al. Indocyaningrün navigierte, roboter assistierte hemihepatekomie links. Zentralbl Chir. (2020). doi: 10.1055/a-1157-9754 and AMa wrote and revised the manuscript as well as acted as the corresponding authors. AMe was the main surgeon and involved directly in managing the patient. All authors contributed to the article and approved the submitted version.
15. Guan R, Chen Y, Yang K, Ma D, Gong X, Shen B, et al. Clinical efficacy of robot-assisted vs. laparoscopic liver resection: a meta analysis. Asian J Surg. (2019) 42:19-31. doi: 10.1016/j.asjsur.2018. 05.008

16. Randell R, Alvarado N, Honey S, Greenhalgh J, Gardner P, Gill A, et al. Impact of robotic surgery on decision making: perspectives of surgical teams. AMIA Annu Symp Proc. (2015) 2015:1057-66.

17. Magistri P, Pecchi A, Franceschini E, Pesi B, Guadagni S, Catellani B, et al. Not just minor resections: robotic approach for cystic echinococcosis of the liver. Infection. (2019) 47:973-9. doi: 10.1007/s15010-019-0 $1333-2$

18. Felsenreich DM, Rojas A, Gachabayov M, Quintero L, Da Eric Dong X. Robotic resection of hepatic echinococcal cysts: a video vignette. Asian J Surg. (2020) 43:857. doi: 10.1016/j.asjsur.2020.04.014

19. Efanov M. Radical robot-assisted liver resection for alveolar echinococcosis. CRSLS MIS Case Rep SLS. (2015) 19:1-4. doi: 10.4293/CRSLS.2015.00021

20. Zhao ZM, Yin ZZ, Meng Y, Jiang $N$, Ma ZG, Pan LC, et al. Successful robotic radical resection of hepatic echinococcosis located in posterosuperior liver segments. World J Gastroenterol. (2020) 26:2831-8. doi: 10.3748/wjg.v26.i21.2831

21. Sato N, Namieno T, Takahashi H, Yamashita K, Matsuhisa T, Aoki S, et al. A long-surviving patient with recurrences of hepatic alveolar echinococcosis after traumatic intra-abdominal rupture. J Gastroenterol. (1996) 31:8858. doi: $10.1007 / \mathrm{BF} 02358620$

22. Guilbaud T, Feretti C, Holowko W, Garbarino GM, Marchese U, Sarran A, et al. Laparoscopic major hepatectomy: do not underestimate the impact of specimen extraction site. World J Surg. (2020) 44:122330. doi: 10.1007/s00268-019-05285-4

23. Teixeira AR, Pilla VF, Makdissi FF, Machado MA. A simple technique for large tumor removal during laparoscopic liver resection. Surg Laparosc Endosc Percutan Tech. (2008) 18:589-91. doi: 10.1097/SLE.0b013e3181809e8d

24. Wang Y, Xiong W, Lan X, Zhang J, Chen T, Liu H, et al. Suprapubic single incision laparoscopic appendectomy. J Surg Res. (2015) 193:57782. doi: $10.1016 /$ j.jss.2014.07.064

25. Zhang Z, Wang Y, Liu R, Zhao L, Liu H, Zhang J, et al. Suprapubic single-incision versus conventional laparoscopic appendectomy. J Surg Res. (2016) 200:131-8. doi: 10.1016/j.jss.2015. 07.032

Conflict of Interest: The authors declare that the research was conducted in the absence of any commercial or financial relationships that could be construed as a potential conflict of interest.

Copyright (C) 2021 Golriz, Flossmann, Ramouz, Majlesara, Kulu, Stojkovic and Mehrabi. This is an open-access article distributed under the terms of the Creative Commons Attribution License (CC BY). The use, distribution or reproduction in other forums is permitted, provided the original author(s) and the copyright owner(s) are credited and that the original publication in this journal is cited, in accordance with accepted academic practice. No use, distribution or reproduction is permitted which does not comply with these terms. 